Hidetoshi Yamada $\cdot$ Atsushi Kunisato

Masahiro Kawahara · Candice G. T. Tahimic

Xianying Ren · Hiroshi Ueda - Teruyuki Nagamune

Motonobu Katoh · Toshiaki Inoue - Mitsuo Nishikawa

Mitsuo Oshimura

\title{
Exogenous gene expression and growth regulation of hematopoietic cells via a novel human artificial chromosome
}

Received: 21 July 2005 / Accepted: 18 October 2005/Published online: 7 December 2005

(C) The Japan Society of Human Genetics and Springer-Verlag 2005

\begin{abstract}
A number of gene delivery systems are currently being developed for potential use in gene therapy. Here, we demonstrate the feasibility of $21 \Delta$ qHAC, a newly developed human artificial chromosome (HAC), as a gene delivery system. We first introduced a $21 \Delta$ qHAC carrying an EGFP reporter gene and a geneticin-resistant gene (EGFP-21 $\Delta$ qHAC) into hematopoietic cells by microcell-mediated chromosome transfer. These HAC-containing hematopoietic cells showed resistance to geneticin, expressed EGFP and retained the ability to differentiate into various lineages, and the EGFP-21 $\Delta$ qHAC was successfully transduced into primary hematopoietic cells. Hematopoietic cells harboring the EGFP-21 $\Delta$ qHAC could still be detected at two weeks post-transplantation in immunodeficient mice. We also showed effective expansion of hematopoietic cells by introducing the $21 \Delta \mathrm{qHAC}$ containing
\end{abstract}

H. Yamada · C.G.T. Tahimic $\cdot$ M. Nishikawa

M. Oshimura $(\square)$

Department of Biomedical Science, Institute of Regenerative Medicine and Biofunction, Graduate School of Medical Science, Tottori University, 86 Nishi-cho, Yonago, Tottori 683-8503, Japan

E-mail: oshimura@grape.med.tottori-u.ac.jp

Tel. + 81-859-348260

Fax: $+81-859-348134$

H. Yamada $\cdot$ X. Ren $\cdot$ M. Katoh $\cdot$ T. Inoue $\cdot$ M. Oshimura Department of Human Genome Science, Graduate School of Medical Science, Tottori University, 86 Nishi-cho,

Yonago, Tottori 683-8503, Japan

A. Kunisato $\cdot$ M. Nishikawa

Pharmaceutical Research Laboratories, Pharmaceutical Division, Kirin Brewery Co., Ltd, 3 Miyahara, Takasaki,

Gunma 370-1295, Japan

M. Kawahara $\cdot$ H. Ueda $\cdot$ T. Nagamune

Department of Chemistry and Biotechnology, Graduate School

of Engineering, The University of Tokyo, 7-3-1 Hongo,

Bunkyo-ku, Tokyo 113-8656, Japan
ScFvg, a gp130-based chimeric receptor that transmits growth signals in response to specific-antigen of this receptor. All of these results demonstrate the usefulness of HAC in gene therapy.

Keywords Human artificial chromosome ·

Hematopoietic cells · Microcell-mediated chromosome transfer $\cdot$ Gene therapy $\cdot$ Chimeric receptor

Abbreviation EGFP: Enhanced green fluorescein protein - HAC: Human artificial chromosome .

MMCT: Microcell-mediated chromosome transfer

hBM MNCs: Human bone marrow mononuclear cells . hCB: Human cord blood

\section{Introduction}

Gene therapy holds tremendous potential for treating diseases. However, each vector system developed for gene therapy so far has advantages and disadvantages, and no "almighty vector system" has been established to address all needs. Here, we describe our novel approach to using chromosomes as vectors for gene delivery. The human artificial chromosome (HAC) system is a realistic choice for clinical gene therapy, because HACs have significant advantages for gene therapy, including a large capacity for DNA inserts, stable extrachromosomal maintenance in host cells and satisfactory gene expression levels (Grimes et al. 2002; Larin et al. 2002; Saffery et al. 2002). We recently developed a novel, centromere-based HAC vector from human chromosome 21 , which referred to as the $21 \Delta \mathrm{qHAC}$ vector. Features of the vector include a single loxP site for sitespecific insertion of DNA via the Cre/loxP system (Kakeda et al. 2005; Katoh et al. 2004). Our group confirmed the stability of HAC with mice bearing HAC possessing the entire human immunoglobin region (Kawahara et al. 2004). 
In this study, we evaluate the feasibility of HAC vectors for gene therapy using primary human bone marrow mononuclear cells (hBM MNC) or cord bloodderived hematopoietic cells.

\section{Materials and methods}

\section{$21 \Delta \mathrm{qHAC}$ vector}

The construction of the $21 \Delta \mathrm{qHAC}$ vector was described in our previous report (Katoh et al 2004). Briefly, the $21 \Delta$ qHAC vector was generated from human chromosome 21 in DT40 cells by removing the distal arm of chromosome $21 \mathrm{q}$ via telomere-directed chromosome truncation. Moreover, we introduced a loxP site between the centromere and the end of 21q, creating a site at which an additional sequence, including genes, can be introduced via the Cre-loxP system. Then the $21 \Delta \mathrm{qHAC}$ vector was transferred from DT40-hybrids to $\mathrm{CHO}$ cells by MMCT. The EGFP-21 $\Delta \mathrm{qHAC}$ vector or ScFvg-21 $1 \mathrm{qHAC}$ is the $21 \Delta$ qHAC vector harboring an hCMV-EGFP gene or a PGK-ScFvg gene (Kawahara et al. 2004) respectively. The ScFvg gene is a chimeric receptor consisting of an anti-fluorescein single-chain, Fv, fused to the extracellular D2 domain of the erythropoietin receptor and the transmembrane/intracellular domains of gp130 (Kawahara et al. 2004). These genes were inserted at the loxP site of the $21 \Delta \mathrm{qHAC}$ vector by Cre-mediated recombination.

Cell culture and microcell-mediated chromosome transfer

Human cord blood cells were obtained from volunteers who had granted their informed consent. Human cell experiments were performed according to the guidelines and with the permission of Kirin Brewery's ethical committee. Both EGFP-21 $\Delta$ qHAC vectors and ScFvg-21 $\Delta$ qHAC vectors retained in $\mathrm{CHO}$ cells were transferred to $1 \times 10^{7} \mathrm{hBM}$ MNCs (AllCells, LLC, Berkeley, CA, USA) or $1 \times 10^{6} \mathrm{hCB} \mathrm{CD} 34^{+}$cells by microcell-mediated chromosome transfer (MMCT) as previously described (Doherty et al. 2003; Kakeda et al. 2005; Katoh et al. 2004). The cells were cultured for five days in the presence of $100 \mathrm{ng} / \mathrm{ml} \mathrm{hSCF}$, $30 \mathrm{ng} / \mathrm{ml} \mathrm{hTPO}$, and $20 \mathrm{ng} / \mathrm{ml}$ FP6 (Kimura et al. 2000; Ueda et al. 2000) or $1 \mu \mathrm{g} / \mathrm{ml}$ BSA-Fl (Sigma, St. Louis, MO, USA). Then the cells were subjected to a clonogenic assay for 12 days in the presence of $50 \mathrm{ng} /$ $\mathrm{ml} \mathrm{SCF}$, $30 \mathrm{ng} / \mathrm{ml}$ TPO, $50 \mathrm{ng} / \mathrm{ml} \mathrm{IL-6,} 50 \mathrm{ng} / \mathrm{ml} \mathrm{IL-3,}$ $50 \mathrm{ng} / \mathrm{ml} \mathrm{GM}-\mathrm{CSF}$, 5IU/ml EPO (Kirin Brewery Co. Ltd., Gunma, Japan), and $800 \mu \mathrm{g} / \mathrm{ml}$ geneticin (Invitrogen, Inchinnan, UK).

\section{PCR analysis of genomic DNA}

For colony-forming cells, the cells picked from methylcellulose culture were subjected to DNA isolation. For FACS-sorted cells, the cells were directly subjected to DNA isolation. The following oligonucleotide primers were used to detect the $21 \Delta \mathrm{qHAC}$ vector: $5^{\prime}$-CGTAACAACTCCGCCCCATT-3' and 5'-CGGACAGGTCGGTCTTGACA-3'. This primer pair specifically detects the loxP-neo region of the $21 \Delta \mathrm{qHAC}$ vector. Genomic DNA was amplified using the following cycle conditions: 5 min at $94{ }^{\circ} \mathrm{C}$ and then 35 cycles of $\left(15 \mathrm{~s}\right.$ at $94{ }^{\circ} \mathrm{C} ; 30 \mathrm{~s}$ at $64{ }^{\circ} \mathrm{C} ; 30$ s at $72{ }^{\circ} \mathrm{C}$ ).

Animals and transplantation

All transplantation experiments were performed following guidelines of the animal ethical committee of Kirin Brewery Co. Ltd. Five-week-old NOD.CB17-Pa$\mathrm{kdc}$ scid/J mice (The Jackson Laboratory, Bar Harbor, ME, USA) were subjected to $200 \mathrm{cGy}$ of X-rays. Cultured hCB CD34 + cells were then injected into the tail vein.

Flow cytometry analysis and cell sorting

For the colony-forming cells, the cells picked from methylcellulose culture were subjected to flow cytometry analysis (BECTON) to detect EGFP expression. The cells taken from the bone marrow of recipient mice two weeks post-transplantation were stained with phycoerythrin-conjugated anti-human CD45 (Pharmingen, San Diego, CA, USA) and allophycocyanin-conjugated anti-mouse CD45 (Pharmingen) and then analyzed by flow cytometry.

\section{$\mathrm{CD}_{3}{ }^{+}$cell selection}

The hCB CD $34^{+}$cells were prepared by MidiMACS (Miltenyi, Auburn, CA, USA). CB CD $34^{+}$cells were labeled with hapten-conjugated monoclonal antibodies against human CD34, followed by anti-hapten antibodies coupled to microbeads. The bead-positive cells $\left(\mathrm{CD} 34^{+}\right.$cells) were enriched on magnetic columns.

\section{Results and discussion}

We previously developed the $21 \Delta \mathrm{qHAC}$ vector that contains a minimized function of chromosomes of centromere and telomere obtained by truncation of chromosome 21. Here, we further explore the potential use of HAC vectors for gene therapy by introducing the $21 \Delta$ qHAC vector to human bone marrow or cord bloodderived hematopoietic cells. We transferred the 
$21 \Delta \mathrm{qHAC}$ vector containing an EGFP transgene and a geneticin resistance marker (EGFP-21 $\triangle \mathrm{qHAC}$ ) from CHO cells to hBM MNCs by MMCT. Subsequently, the cells were cultured for five days and then subjected to a clonogenic assay in the presence of geneticin. The mean number of geneticin-resistant colonies was $53 \pm 15.5$ (standard deviation). Among them, the mean number of EGFP-positive colonies was $50 \pm 16.3$ (standard deviation). The average transfer efficiency was $4.0 \times 10^{-4}$, corresponding to a total of 53 geneticin-resistant colonies (20 granulocyte-macrophage colonies and 33 erythroid colonies) per $2.6 \times 10^{5}$ colony-forming cells (Fig. 1a). We confirmed that the cells expressing EGFP actually carried the EGFP-21 $1 \mathrm{qHAC}$ by PCR. We observed expected size of PCR product for loxP-neo, suggesting that the $21 \Delta \mathrm{qHAC}$ vector is maintained in these cells (Fig. 1b). FACS analyses revealed that $72 \%$ of the cells in those colonies expressed EGFP (Fig. 1c). The presence of the cells that do not express EGFP might be explained by the gradual loss of HAC vectors through continuous cell division or chromosome inactivation (Katoh et al 2004). A fluorescence in situ hybridization analysis to detect the $21 \Delta \mathrm{qHAC}$ vector was not successful due to technical difficulties with obtaining metaphase spreads of chromosome. Although we failed to demonstrate the independent existence of HAC in this study, other studies have shown that the $21 \Delta$ qHAC vector is maintained as an independent chromosome and is mitotically stable in human cell lines, primary human fibroblasts and human mesenchymal stem cells (Kakeda et al. 2005; Katoh et al.2004; Ren et al. 2005). This observation supports the idea that the $21 \Delta \mathrm{qHAC}$ vector is mitotically stable in human cells. May-Giemsa staining revealed that EGFP-expressing colonies contained not only erythroid cells but also various types of differentiated blood cells such as macrophages and primitive erythrocyte, in agreement with the results of the clonogenic assay (Fig. 1d). These results proved that satisfactory transfer efficiencies and remarkably high percentages of transgene expression are obtained, and that the proper ability to successful differentiate into a variety of cell lineages is retained.

Next, we evaluated the in vivo repopulating potential of hematopoietic cells carrying the HAC by xenogenic transplantation. We introduced the EGFP-21 $\Delta$ qHAC vector into purified cord blood-derived CD34-positive

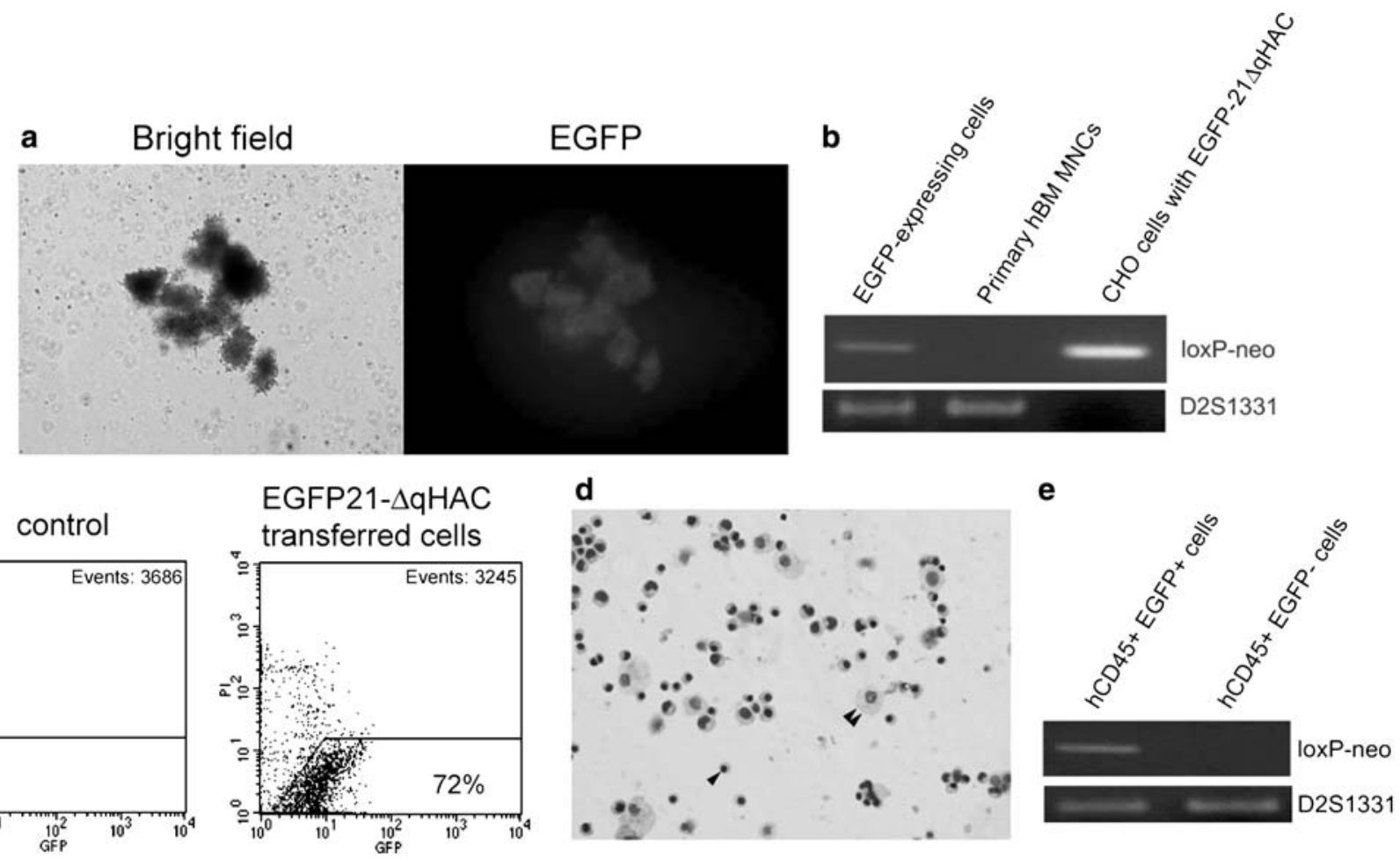

Fig. 1a-e $21 \Delta$ qHAC facilitates gene transfer to human hematopoietic cells. a Morphology of an EGFP-expressing colony-expressing burst-forming unit of erythroid. Representative fluorescence and phase contrast microscopic view of geneticinresistant colony. Original magnification: $\times 100$. b PCR detection of the $21 \Delta$ qHAC vector in EGFP-positive cells. Genomic DNA from $\mathrm{CHO}$ cells was used as a positive control. It is noted that the amount of template DNA is extracted from only one colony (about 300 cells) of "EGFP expressing cells" and "primary hBM MNCs". On the other hand, DNA extracted from "CHO" cells is from many more than 300 cells. D2S1331 is a STS marker on human chromosome 2. c FACS profiles of the cells from control colonies (left panel) and EGFP-21 $\Delta \mathrm{qHAC}$-containing colonies (right panel). 3,687 cells and 3,245 cells are analyzed, respectively. d May-Giemsa staining of EGFP-positive colony-forming cells. An arrowhead and a double arrowhead indicate typical images of primitive erythrocyte and macrophage, respectively. Images were taken at $400 \times$ magnification. e PCR analysis of the human cells isolated from the representative recipient mice after two weeks posttransplantation 
Table 1 Selective growth of human hematopoietic cells with ScFvg-21 $\Delta$ qHAC

\begin{tabular}{lcccccc}
\hline & Exp. 1 & Exp. 2 & Exp. 3 & Exp. 4 & Exp. 5 & Average \\
\hline BSA-Fl(-) & 13 & 7 & 3 & 16 & 18 & 11.4 \\
BSA-Fl(+) & 34 & 35 & 13 & 78 & 92 & 50.4 \\
Expansion factor $^{\mathrm{a}}$ & 2.6 & 5 & 4.3 & 4.8 & 5.1 & $4.3 \pm 1.0$ \\
\hline
\end{tabular}

Total number of geneticin-resistant colonies. After 12 days in culture, a total of $11.4 \pm 6.2$ or $50.4 \pm 33.1$ geneticin-resistant colonies were obtained in the absence or presence of BSA-Fl, respectively. The total number of geneticin-resistant colonies increased by a factor of $4.36 \pm 1.0$ with BSA-Fl treatment

${ }^{\mathrm{a}} \mathrm{BSA}-\mathrm{Fl}(+) / \mathrm{BSA}-\mathrm{Fl}(-)$

hematopoietic stem cells (hCB $\mathrm{CD} 34^{+}$) via MMCT. These cells were cultured for five days in the presence of hSCF, hTPO, and FP6, and intravenously injected into sublethally irradiated NOD-SCID mice. Two weeks after transplantation, we harvested bone marrow cells and counted the cells that were positive for EGFP and hCD45 (human blood cell marker). Out of $1.2 \times 10^{4}$ hCD45-positive cells, 18 were found to be positive for EGFP. A PCR-based analysis revealed that HAC-derived PCR product was detected for $\mathrm{EGFP}^{+}$, hCD45 ${ }^{+}$cells, but not for $\mathrm{EGFP}^{-}, \mathrm{hCD}^{+} 5^{+}$cells (Fig. 1e). These results demonstrate that cells carrying a HAC could still be observed in immunodeficient mice at two weeks post-transplantation.

Finally, we examined the possibility of expanding hematopoietic cells by introducing a gene conferring a growth advantage via our HAC system. We used ScFvg, a gp130-based chimeric receptor that transduces a growth signal in response to a ligand, fluorescein-conjugated BSA (BSA-Fl). The ScFvg-21 $\Delta \mathrm{qHAC}$ was introduced into hBM MNCs via MMCT and the cells were cultured for five days in the presence or absence of BSA-Fl prior to the clonogenic assay. There were $11.4 \pm 6.2$ geneticin-resistant colonies in the untreated set and $50.4 \pm 33.1$ geneticin-resistant colonies in the set treated with BSA-Fl (Table 1). The number of colonies was not altered by the addition of BSA-Fl in normal hBM MNCs (data not shown). The total number of geneticin-resistant colonies increased by a factor of $4.3 \pm 1.0$ with BSA-Fl. These data demonstrate that the ScFvg gene carried by the $21 \Delta \mathrm{qHAC}$ vector was functional in hBM MNCs, and that it conferred a growth advantage to cells upon treatment with BSA-Fl.

We recently reported on the transfer of a HAC to primary human fibroblasts (Kakeda et al. 2005). To our knowledge, this is the second report of a successful transfer of a HAC into primary human cells and the first report of a successful transfer of a HAC into primary human hematopoietic cells, transplantation, and retention of these cells in bone marrow. Taken together, our results show the promise of HACs as tools for gene therapy. Our HAC-based system also provides a model for therapeutic gene delivery that is compatible with current clinical procedures such as bone marrow transplantation. Further improvements to HAC vector design and gene delivery systems to meet stringent clinical requirements will involve further study.
Acknowledgements We thank Drs. K. Tomizuka, M. Kakeda, and M. Hiratsuka for valuable discussions. This study was supported in part by a Health and Labour Sciences Research Grant for Research on Human Genome, Tissue Engineering from the Ministry of Health, Labour and Welfare, Japan and by the Twenty-First Century COE Program of the Ministry of Education, Culture, Sports, Science and Technology of Japan: The Research Core for Chromosome Engineering Technology.

\section{References}

Doherty AM, Fisher EM (2003) Microcell-mediated chromosome transfer (MMCT): small cells with huge potential. Mamm Genome 14:583-592

Grimes BR, Warburton PE, Farr CJ (2002) Chromosome engineering: prospects for gene therapy. Gene Ther 9:713-718

Kakeda M, Hiratsuka M, Nagata K, Kuroiwa Y, Kakitani M, Katoh M, Oshimura M, Tomizuka K (2005) Human artificial chromosome (HAC) vector provides long-term therapeutic transgene expression in normal human primary fibroblasts. Gene Ther 12:852-856

Katoh M, Ayabe F, Norikane S, Okada T, Masumoto H, Horike S, Shirayoshi Y, Oshimura M (2004) Construction of a novel human artificial chromosome vector for gene delivery. Biochem Biophys Res Commun 321:280-290

Kawahara M, Kimura H, Ueda H, Nagamune T (2004) Selection of genetically modified cell population using hapten-specific antibody/receptor chimera. Biochem Biophys Res Commun 315:132-138

Kimura T, Wang J, Minamiguchi H, Fujiki H, Harada S, Okuda $\mathrm{K}$, Kaneko H, Yokota S, Yasukawa K, Abe T, Sonoda Y (2000) Signal through gp130 activated by soluble interleukin (IL)-6 receptor (R) and IL-6 or IL-6R/IL-6 fusion protein enhances ex vivo expansion of human peripheral blood-derived hematopoietic progenitors. Stem Cells 18:444-452

Kuroiwa Y, Tomizuka K, Shinohara T, Kazuki Y, Yoshida H, Ohguma A, Yamamoto T, Tanaka S, Oshimura M, Ishida I (2000) Manipulation of human minichromosomes to carry greater than megabase-sized chromosome inserts. Nat Biotechnol 18:1086-1090

Larin Z, Mejia JE (2002) Advance in human artificial chromosome technology. Trends Genet 18:313-319

Ren X, Katoh M, Hoshiya H, Kurimasa A, Inoue T, Ayabe F, Shibata K, Toguchida J, Oshimura M (2005) A novel human artificial chromosome vector provides effective cell lineagespecific transgene expression in human mesenchymal stem cells. Stem Cells (in press)

Saffery R, Choo KH (2002) Strategies for engineering human chromosomes with therapeutic potential. J Gene Med 4:5-13

Ueda T, Tsuji K, Yoshino H, Ebihara Y, Yagasaki H, Hisakawa $\mathrm{H}$, Mitsui T, Manabe A, Tanaka R, Kobayashi K, Ito M, Yasukawa K, Nakahata T (2000) Expansion of human NOD/ SCID-repopulating cells by stem cell factor, Flk2/Flt3 ligand, thrombopoietin, IL-6, and soluble IL-6 receptor. J Clin Invest 105:1013-1021 